\title{
Rear Infrared Thermography in Heat Fluxes Determination on Hypersonics Vehicles
}

\author{
by Anne Hoornaert*, Christian Pelissier*, Yves Le Sant**, Frédéric Thivet* \\ and Pierre Millan* \\ * ONERA DMAE/C2A TOULOUSE FRANCE \\ ** ONERA DAFE/IMOS MEUDON FRANCE
}

\begin{abstract}
Due to agressive external conditions during an hypersonic flight, parameters such as external wall temperature are not easily measurable with the usual approach. Hence this study develops a promising in flight measurements methodology to estimate heat fluxes on space vehicle external surface, in order to improve the aerodynamic models. One important application will be the design of the thermal protections. THEFA technique consists in using rear infrared thermography measurements and an unsteady inverse heat transfer model ([1]). The aim is to determine the heat fluxes received by the external surface of the vehicle by using the temporal evolution of its internal face thermal map.
\end{abstract}

\section{Introduction}

This study is motivated by the growing interest in the design of Reusable Launch Vehicles (RLV). Critical elements of the design are the flaps and Thermal Protection System (TPS) because their sizing dramatically depends on hypersonic flow aerothermodynamic phenomena (real gas effects, wall catalycity, laminary/turbulent transition...). Due to the lack of accurate knowledge of these phenomena, the usual conservative assumptions on TPS are often penalizing for the payload. Therefore it is necessary to characterize in-flight critical parameters such as pressure and wall heat fluxes distributions. Due to the limits of the onground validation, a large interest goes to in-flight measurements. Because of agressive flow conditions during a hypersonic flight, external wall heat flux measurements are difficult, often impossible, particularly in regions where high heat flux levels are created by phenomena such as transition and interaction.

The purpose of this study is to develop and validate an innovative methodology called THEFA ("THermographie en Face Arrière" in french which is a translation of "Rear Face Thermography") to determine heat fluxes received by the external wall of hypersonic vehicles. The THEFA technique consists in using rear infrared thermography measurements and an unsteady inverse heat transfer model. The first step of the onground validation requires the design of a test model. Our choice, based on previous studies [2], is a double-cone model. This configuration allows to obtain an interaction between the boundary layer which develops from the first-cone nose, and the second shock on the larger cone. In some conditions, this interaction causes the separation of the boundary layer and generates over-fluxes, as illustrated in figure 1. 


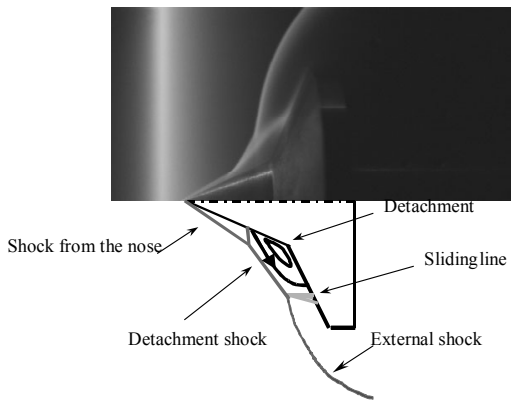

Figure 1: Flow visualization by FFE and flow pattern around the double cone model from [2].

\section{Numerical method}

The inverse heat conduction problem consists in determining the external heat fluxes from temperature measurements on the back side of the wall. This is an illposed problem which is not as easy to solve as the direct conduction problem. Due to the measurement errors, the inversion method cannot use the perfect equality between direct model and experimentation. There are two kinds of technique based on minimization:

-whole domain resolution

-sequential resolution

The whole domain method proposed by Tikhonov [3] consists in minimizing in one step the difference between measured and estimated temperatures at every time on the whole domain:

$$
R(q)=\sum_{i=1}^{N m e s} \sum_{t=1}^{N t}\left(Y_{i}^{t}-T_{i}^{t}(\vec{q})\right)^{2}
$$

However, this technique is very time-consuming especially in threedimensional cases.

Another approach proposed by Beck ([4]) consists in minimizing sequentially the same kind of functional. As a temporal stabilization procedure, the heat flux is assumed to be constant or linear for $r$ future temperatures. At each time $n$, the functional is:

$$
R(q, n)=\sum_{i=1}^{N m e s} \sum_{t=0}^{r-1}\left(Y_{i}^{n+t}-T_{i}^{n+t}(\vec{q})\right)^{2}
$$

From the experimental rear infrared measurements, we get thermal internal surface history. A numerical code based on Beck's inverse algorithm [4] allows to obtain the external fluxes.

\section{Test campaign in $\mathrm{R} 5 \mathrm{Ch}$ wind tunnel}

\subsection{Design of the experimental program}

The geometry, recently tested in ONERA [2], is composed of two axisymetric cones, with angles of $25^{\circ}$ and $65^{\circ}$ respectively. The model, used in R5Ch wind tunnel, has small dimensions (60 mm diameter) and its thickness is $3 \mathrm{~mm}$ (figure 2). Downstream the second cone, a piece of cylinder was added with a diameter equal to $60 \mathrm{~mm}$. 


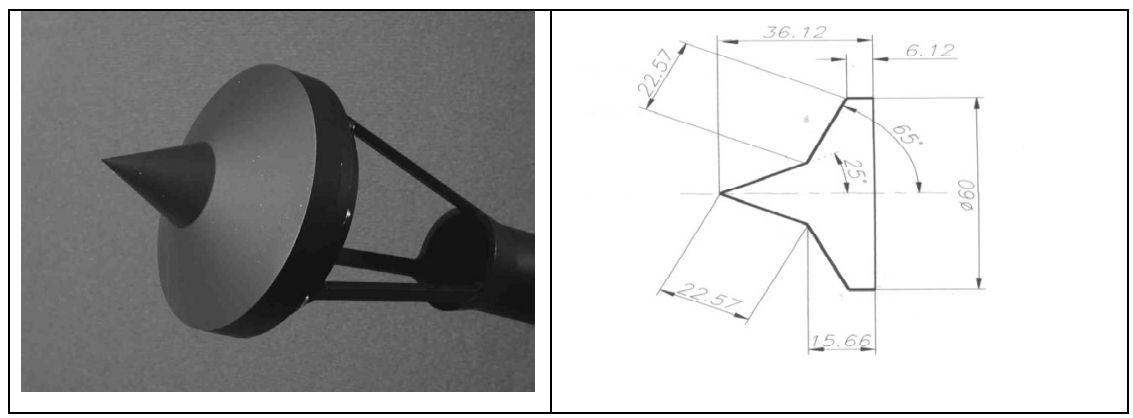

Figure 2: The double cone model (dimensions in $\mathrm{mm}$ ).

The material of the model is ISOTAN $^{\odot}$, the characteristics of which are gathered in Table 1.

Table 1: ISOTAN thermal properties.

\begin{tabular}{|c|c|c|c|c|}
\hline $\begin{array}{c}\text { Temperature } \\
\left({ }^{\circ} \mathrm{C}\right)\end{array}$ & $\begin{array}{c}\text { Diffusivity } \\
\left(10^{-6} \mathrm{~m}^{2} / \mathrm{s}\right)\end{array}$ & $\begin{array}{c}\text { Thermal } \\
\text { capacity } \\
\left(\mathrm{J} . \mathrm{K}^{-1} \cdot \mathrm{kg}^{-1}\right)\end{array}$ & $\begin{array}{c}\text { Density } \\
\left(\mathrm{kg} / \mathrm{m}^{3}\right)\end{array}$ & $\begin{array}{c}\text { Conductivity } \\
\left(\mathrm{W} . \mathrm{m}^{-1} . \mathrm{K}^{-1}\right)\end{array}$ \\
\hline 23 & 6.00 & 408 & 8852 & 21.7 \\
\hline 50 & 6.22 & 421 & 8842 & 23.2 \\
\hline
\end{tabular}

The first step is to validate the THEFA technique in steady flow conditions. In wind-tunnel R5Ch over-fluxes generated by shock boundary layer interaction have been observed with infrared thermography measurements on front and back wall faces.

\section{2. $\quad$ Test facilities}

\subsubsection{The R5Ch blow-down wind tunnel}

The aerodynamic circuit is composed of a Joule effect heater, a nozzle, a testing chamber, a diffuser and a vacuum sphere. The flow conditions are:

-Blowdown duration: up to $90 \mathrm{~s}$

-Free test section equipped with a Mach 10 (exit diameter $0.350 \mathrm{~m}$ )

-Variable stagnation pressure: $\mathrm{Pi}=2.5$ bar

-Stagnation temperature: $1000 \mathrm{~K}$ (by instantaneous joule-effect heater)

-Unit Reynolds number in free flow: $167,000 \mathrm{~m}^{-1}$

The R5Ch wind tunnel is designed for investigating hypersonic flows in continuous molecular regime at very low Reynolds numbers (simulating fully laminar flows).

\subsection{2. $\quad$ Test assembling}

The model location in the testing chamber allows to visualize simultaneously the front and the back face of the second cone with the cameras. The 'front' camera was located in the testing chamber in a pressurized box. The 'rear' IR camera was outside the chamber, visualizing the model through an infrared window (transmission of 0.97 ). 


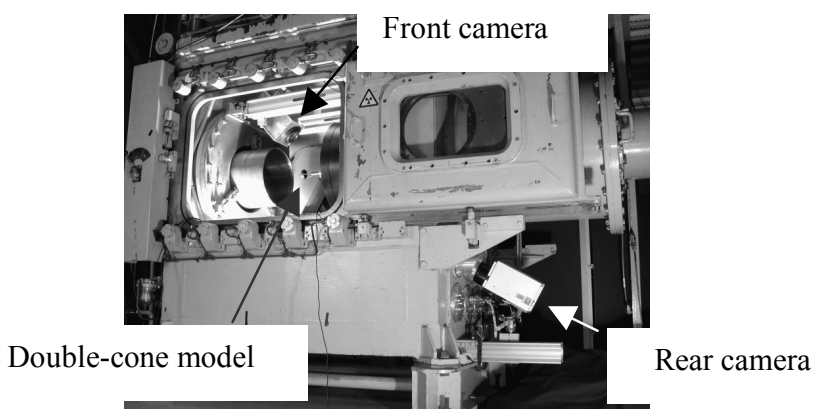

Figure 3: The test assembling.

\subsubsection{Instrumentation}

The 'front' camera is a FLIR IR Systems type SC500, band III (8-12 $\mu \mathrm{m})$ and provides images of $320^{\star} 240$ pixels. The horizontal field of view is $24^{\circ}$. The temperature resolution is $70 \mathrm{mK}$. The 'rear' camera is a CEDIP Jade LW $(8-12 \mu \mathrm{m})$ and provides images of $320^{\star} 240$ pixels. Two lens were used : one of $50 \mathrm{~mm}$ (field of view $11^{\circ *} 8.2^{\circ}$ ) and one of $100 \mathrm{~mm}$ (field of view $5.5^{\circ *} 4^{\circ}$ ). The temperature resolution is $23 \mathrm{mK}$.

Different calibration tests were conducted in-situ at the beginning and during the campaign test. The objective is to prevent from small temporal drift and from transmissivity changes of the IR windows. A black body was installed in front of the cameras to obtain calibration laws, which are polynomial functions (order 1 for FLIR camera, order 4 for CEDIP camera). Each camera was operated through a PC and the recording rate was 50 images/second. The two cameras were triggered.

\section{Results}

Some examples of infrared measurement pictures from the THEFA R5Ch campaign are presented below (figure $4 \& 5$ ).

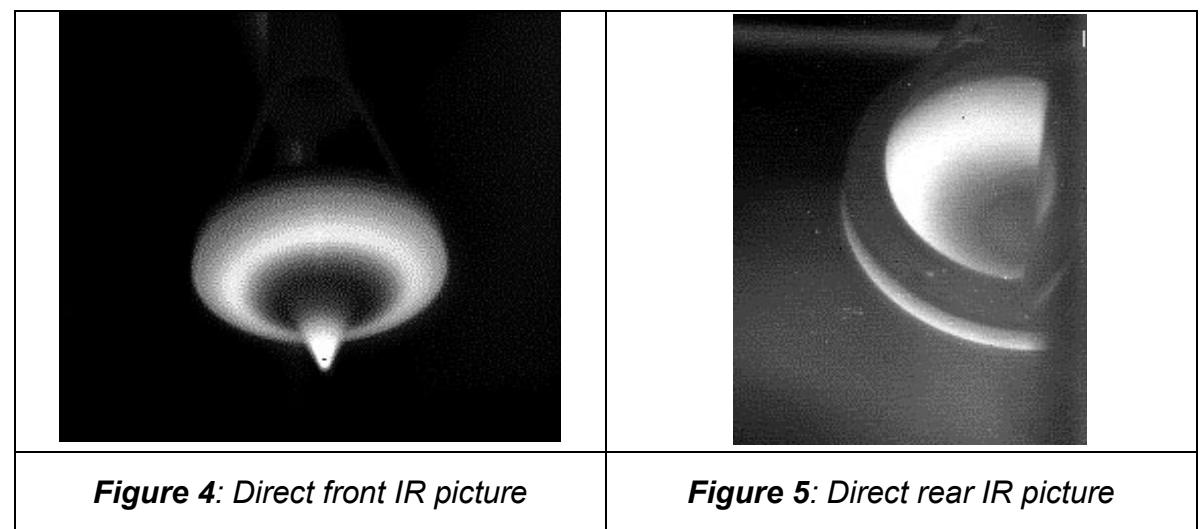

IR total acquisition times are $6.5 \mathrm{~s}$ on the front face and $10 \mathrm{~s}$ on the back face. The experimental acquisition frequency is $50 \mathrm{~Hz}$ and one image out of five is used to run the inverse computation. Since the full 3D inverse method is currently under development, the planar 3D inverse method is used in present analysis. The IR 
analysis of one part of the second cone (figure 6) allows to obtain the temperature temporal evolutions. The front heat flux images (figure 7) are calculated and straightened with the ONERA model FLUX1D [5].

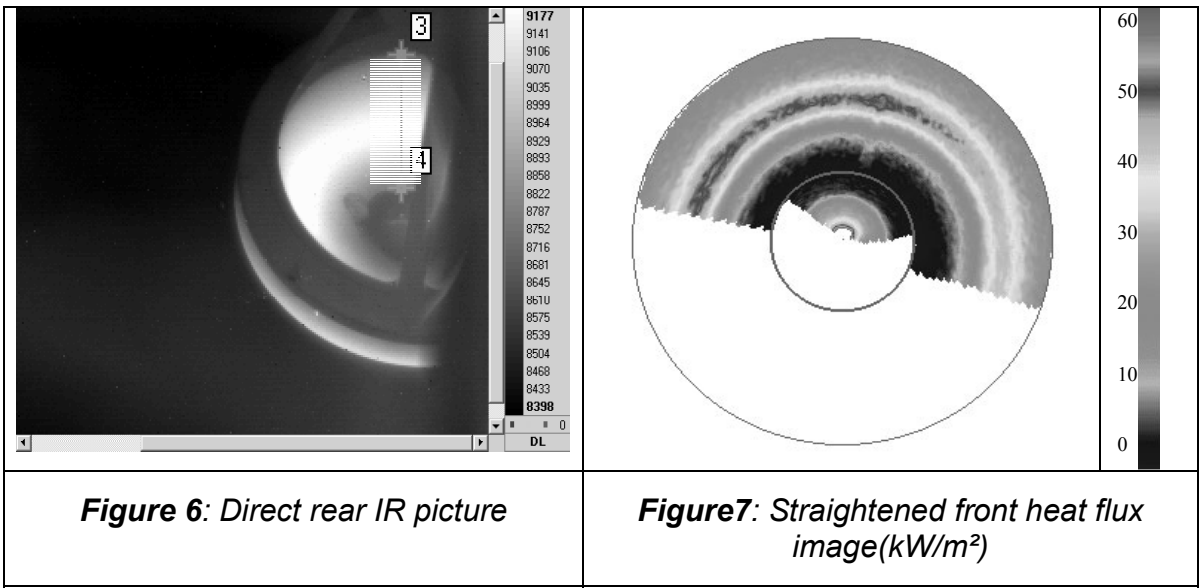

A Butterworth filter (order 6) is used to stabilize temporally the temperature evolution and a 2D median filter allows to smooth spacially the temperature distribution (figure 8). As the flow is axisymetric, the 3D computation results are only shown along a generatrix of the model and at the center of the extracted line. From these internal filtered temperature distribution evolution, the inverse model computes the external one (figure 9) which is compared to the IR front measurements.

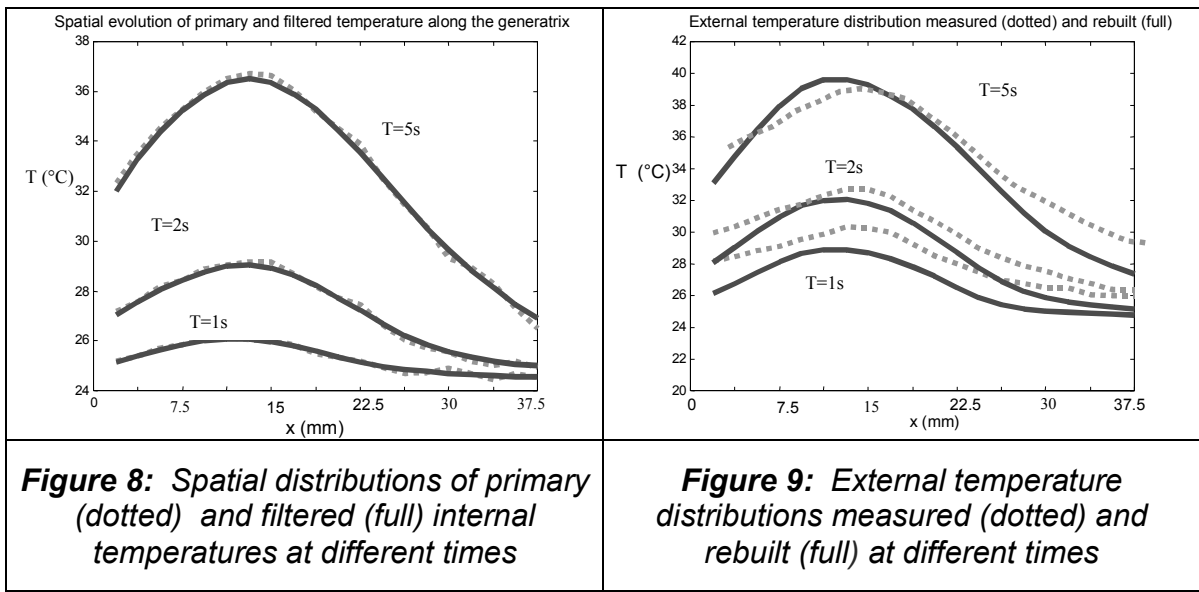

The front heat fluxes distribution is computed by the 3D Beck's inverse model [1] on the chosen area (figure 6) and is in good agreement with the measured one taking into account that the rebuilt procedure neglects the surface curvature of the model. The resulting fluxes along the generatrix and at the hottest point $(x=$ $11.25 \mathrm{~mm}$ ) are shown in figures $10 \& 11$. 


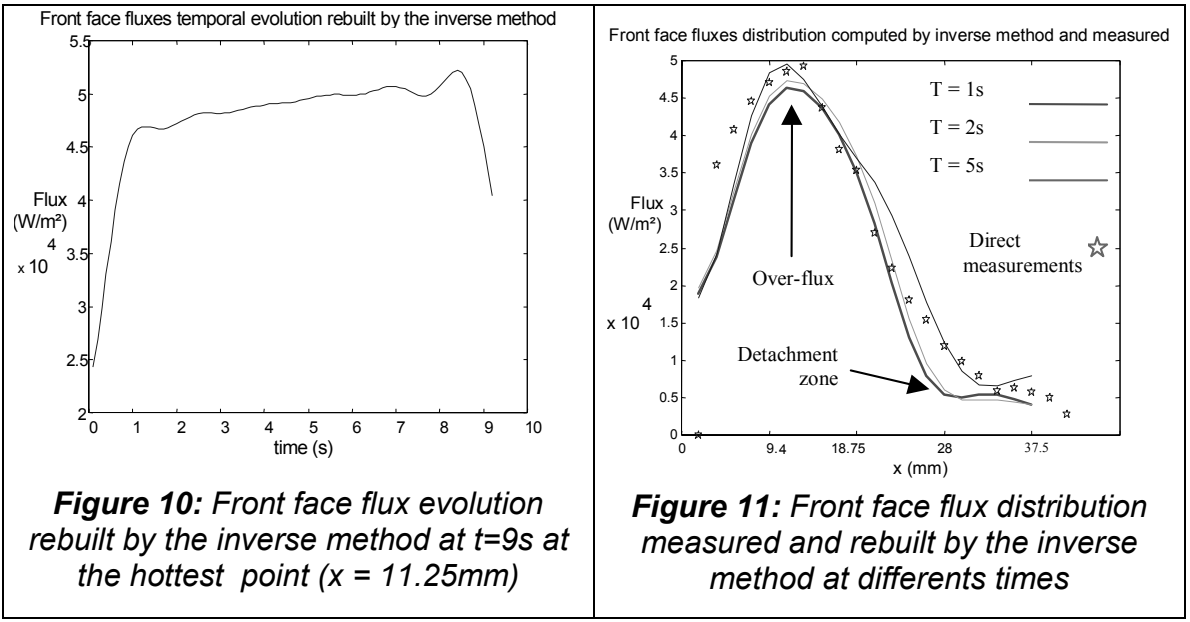

The temporal heat flux assessment is representative of the R5Ch wind-tunnel flow condition. The heat flux computed distribution is in good agreement with the front measurement and with the considered flow distribution (figure 1) taking into account that the rebuilt procedure neglects the surface curvature of the model. The different flow regions are identified in the rebuilt heat flux distribution.

\section{Conclusion}

Tests have been successfully conducted in the R5Ch wind-tunnel to obtain IR measurements of the heat fluxes both on the front and back faces of a double-cone model. The obtain axisymetric flux distribution is representative of the in-flight flux condition. The heat fluxes received by the external surface of the double-cone model were determined with the 3D ONERA unsteady inverse heat transfer method by using the temporal evolution of its internal face thermal map. The flux distribution and temperature evolution rebuilt on the front face with the inverse method is in good agreement with direct external measurements. Planned future activities are the full 3D inverse method development (including curvature effects) and the THEFA validation in the F4 wind-tunnel to verify the ability of the method to determine highly unsteady fluxes and real gas effects.

\section{REFERENCES}

[1] D. NORTERHAUSER \& P. MILLAN, «Resolution of three-dimensionnal unsteady inverse problem by sequential method using parameter reduction and infrared thermography measurements », Numerical Heat Transfer, Part A, Vol. 37, pp 587-611, 2000.

[2] B. CHANETZ, T. POT, R. BENAY, J. MOSS, « New test-cases in Low density hypersonic flow », $23^{\text {rd }}$ Rarefied Gas Dynamics Conference, Whistler, Canada, July 20-25, 2002.

[3] A.N. TIKHONOV \& V. Y. ARSENIN, "Solutions of ill-posed problems », V. H. Winston and Sons, Washington D.C., 1977.

[4] J.V. BECK, B. BLACKWELL \& C. ST CLAIR, "Inverse heat conduction: ill posed problems », Wiley Interscience, New York, 1985.

[5] Y. LE SANT, M. MARCHAND, P. MILLAN and J. FONTAINE, "On overview of infrared thermography techniques used in large wind tunnels", Aerospace Science and Technology, Vol. 6, Issue 5, p 355-366, September 2002 . 